\title{
Erratum de: Description de deux espèces nouvelles du genre Lychnus (Mollusca, Gastropoda, Anadromidae) au Rognacien (Maastrichtien supérieur) de Vitrolles (Bouches-du-Rhône, France)
}

\section{BSGF - Earth Sciences Bulletin 2017, 188, 38 https://doi.org/10.1051/bsgf/2017204}

Guy Turin*

rue de la Mairie, 77320 Saint-Martin-du-Boschet, France

Accepté le 18 janvier 2018

Une version non finalisée de cet article a été publiée. Les éditeurs et le service de publication tiennent à s'excuser auprès de l'auteur et des lecteurs, faisant suite à un problème inédit survenu dans le nouveau processus de mise en ligne des articles.

Vous trouverez ci-dessous la version corrigée.

\footnotetext{
*Auteur correspondant : turin.guy77@orange.fr
} 


\title{
Description de deux espèces nouvelles de Lychnus (Mollusca, Gastropoda, Anadromidae) du Rognacien (Maastrichtien supérieur) de Vitrolles (Bouches-du-Rhône, France)
}

\author{
Guy Turin* \\ 8 rue de la Mairie, 77320 Saint-Martin-du-Boschet, France
}

\begin{abstract}
Résumé - Sur la commune de Vitrolles (Bouches-du-Rhône), le Rognacien (Maastrichtien supérieur) calcaire surplombant la partie orientale de l'Étang de Berre comprend principalement la barre calcaire de Rognac (Rognacien moyen) avec des niveaux marneux intermédiaires, suivi de $12 \mathrm{~m}$ au-dessus d'une cuesta calcaire attribuée au Rognacien supérieur. Le genre Lychnus comprend dans ce Rognacien terminal deux espèces Lychnus matheroni Requien, 1842 et $L$. vitrollensis Répelin, 1920 ; ces taxons sont accompagnés par deux nouvelles espèces Lychnus siruguei nov. sp. et Lychnus applanatus nov. sp. Ces deux nouvelles espèces font l'objet de la présente publication.
\end{abstract}

Mots clés : vitrolles / Rognacien supérieur / Maastrichtien / Lychnus / nouvelles espèces

\begin{abstract}
Description of two new species on the genus Lychnus (Mollusca, Gastropoda, Anadromidae) the Rognacien (upper Maastrichtian) of Vitrolles (Bouches-du-Rhône, French). On the commune of Vitrolles territory (Bouches-du-Rhône), the calcareous Rognacian (upper Maastrichtian) above the eastern part of the Étang de Berre consists mainly of the Rognac calcareous bar (middle Rognacian) with intermediary marly layers topped $12 \mathrm{~m}$ above by a calcareous cuesta attributed to the upper Rognacian. The genus Lychnus consists in this terminal Rognacian of two species Lychnus matheroni Requien, 1842 and L. vitrollensis Répelin, 1920; these taxa are accompanied by two other new species Lychnus siruguei nov. sp. and Lychnus applanatus nov. sp. The description of these two new species is the subject of this publication.
\end{abstract}

Keywords: vitrolles / upper Rognacian / Maastrichtian / Lychnus / new species

\section{Introduction}

Si la présence du genre Lychnus en Provence est connue depuis le $\mathrm{XIX}^{\mathrm{e}}$ siècle (Matheron, 1832), il est certain qu'à l'exception de travaux plus récents (Répelin, 1920; FabreTaxy, 1959), le complément du matériel récolté n'a pratiquement jamais fait l'objet de publications paléontologiques comportant des descriptions, coupes et photographies. Le présent travail consiste à enrichir la biodiversité de ce genre en décrivant deux nouvelles espèces dans le Rognacien supérieur (Maastrichtien supérieur). Jusqu'à maintenant, aucune espèce du genre Lychnus n'a été récoltée dans les couches sus-jacentes de la partie sommitale du calcaire de Rognac (Rognacien moyen). Les collections du Muséum d'Histoire Naturelle de Marseille et celles du MNHN de Paris sont dépourvues également de ces nouvelles espèces.

\footnotetext{
*Auteur correspondant : turin.guy77@orange.fr
}

\subsection{Cadre géographique}

Les espèces nouvelles Lychnus siruguei nov. sp. et $L$. applanatus nov. sp. ont été récoltées sur la commune de Vitrolles (Fig. 1), au chemin du Trou du Loup, aux coordonnées: $\mathrm{N}$ 43. 469092, E 5. 243445, dans un banc calcaire carbonaté $\left(\mathrm{n}^{\circ} 100\right.$, Fig. 2$)$. Ce niveau se prolonge au sud, vers le Griffon et la commune des Pennes-Mirabeau, vers le nord on le retrouve à Saragousse sur la commune de Rognac. Cet horizon calcaire est totalement absent dans le Rognacien supérieur de la partie orientale du bassin d'Aix (Dughi et Sirugue, 1958).

\subsection{Lithologie et biostratigraphie}

Les deux nouvelles espèces ont été trouvées $12 \mathrm{~m}$ audessus du sommet de la cuesta du calcaire de Rognac (Rognacien moyen), sur la partie sommitale d'une barre 


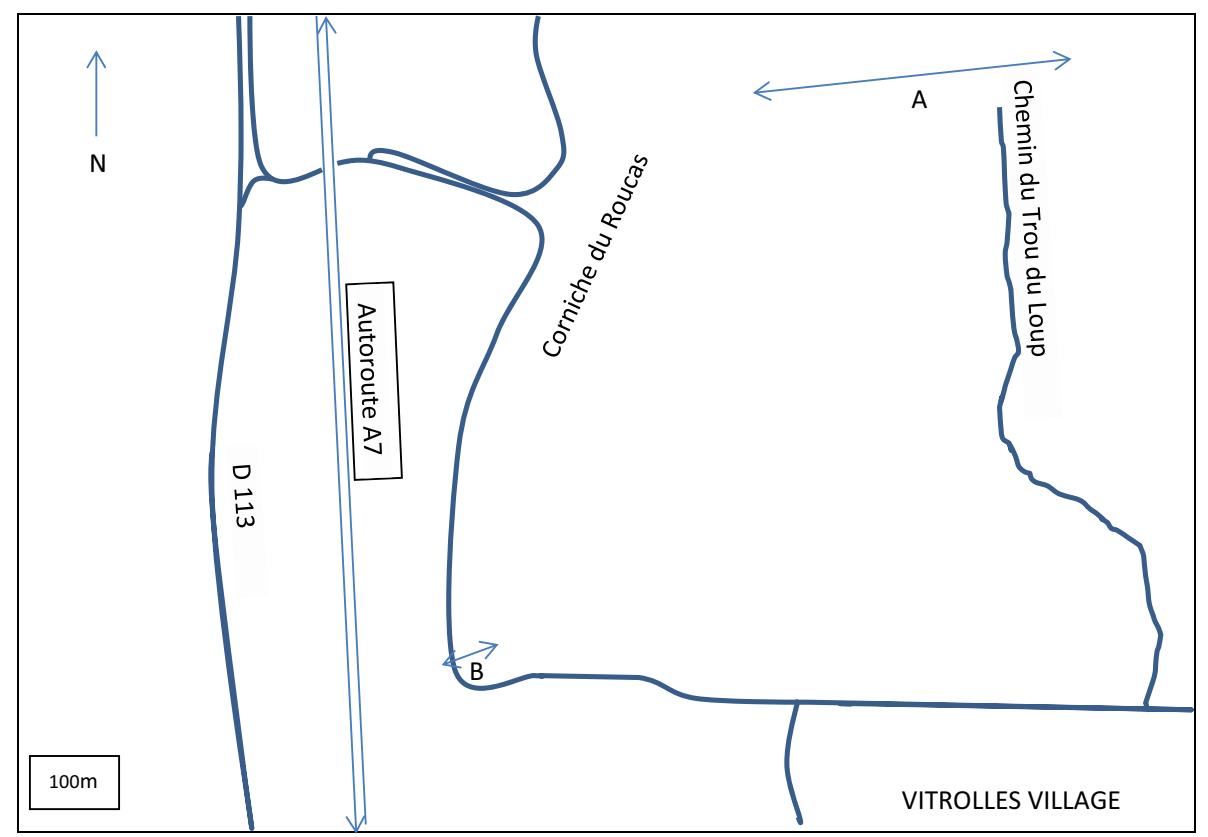

Fig. 1. Localisation des deux coupes A et B sur la commune de Vitrolles (Bouches-du-Rhône).

calcaire de $4 \mathrm{~m}$ d'épaisseur. Celle-ci est constituée sur les deux premiers mètres d'une zone de calcaire gris marneux sans fossiles de gastéropodes, suivie sur les deux mètres suivant d'un calcaire carbonaté rose comprenant les espèces décrites dans le tableau de la coupe A (Fig. 2). L'horizon sus-jacent est composé de $0,5 \mathrm{~m}$ de calcaire marneux rose, c'est le dernier banc fossilifère qui a livré un Lychnus matheroni Requien, 1842, du Maastrichtien supérieur de la série fluvio-lacustre et continentale de Vitrolles.

En ce qui concerne le Rognacien moyen, une coupe B a été réalisée sur la corniche du Roucas (Fig. 3), elle comprend la totalité du calcaire de Rognac avec des niveaux marneux et ligniteux intermédiaires. Ces bancs de calcaire lacustre font 22,5 m d'épaisseur sur la totalité, la série ici est beaucoup plus mince que sur la commune de Rognac (Durand, 1978), cette matière massive de couleur gris blanc est composée de Chara Linnaeus, 1753 et de Cyanophycées, les horizons marneux déposés entre-deux représentent les périodes d'assèchement d'un ancien lac. Le genre Lychnus est présent surtout dans la partie sommitale de cette série, les espèces récoltées sont les suivantes: Lychnus vidali Répelin, 1920, L. urgonensis Matheron, 1842, (synonyme plus ancien de L. bourguignati Munier-Chalmas, 1885), L. matheroni Requien, 1842, L. ellipticus Matheron, 1832 et L. rimatus Matheron in Répelin, 1920. J'ai également récolté un exemplaire de L. pradoanus Verneuil et Lartet, 1863 dans un horizon ligniteux sous-jacent.

Le matériel étudié dans le niveau du Rognacien supérieur est beaucoup plus réduit que dans la série du calcaire de Rognac, il comprend: Palaeocyclophorus sp., Viviparus deshayesi Matheron, 1842, Bauxia bulimoides Matheron, 1832 et le genre Lychnus. Parmi ce dernier, seules les formes carénées sont présentes, le genre Lychnus ayant fait son apparition au Valdonien (Campanien) avec l'espèce L. elongatus Roule, 1885 disparaîtra complètement au-dessus du banc $\mathrm{n}^{\circ} 101$ (Fig. 2), les formes arrondies ne se récoltent que dans les assises du calcaire de la gare de Rognac qui datent du Rognacien inférieur et également au sommet du Rognacien moyen (calcaire de Rognac).

Les gastéropodes fluvio-lacustres et continentaux sont mélangés par lessivage dans la partie sommitale du banc $\mathrm{n}^{\circ} 100$, celui-ci nous a fourni un échantillonnage de quatre espèces de Lychnus carénés qui sont : L. matheroni Requien, 1842, L. vitrollensis Répelin, 1920, L. siruguei nov. sp. et $L$. applanatus nov. sp. L'holotype de Lychnus siruguei nov. sp. reste dans l'ensemble en bon état avec la particularité de posséder encore un peu de coquille, il peut donc servir de diagnose dans le texte suivant, quant à l'holotype et le paratype de Lychnus applanatus nov. sp., malgré le bon état général des spécimens, les tours juvéniles restent érodés.

\section{Systématique}

Famille : Anadromidae Wenz, 1940

Genre : Lychnus Matheron, 1832

Espèce-type: Lychnus ellipticus Matheron, 1832

Lychnus siruguei nov. sp. Pl., fig. A-D.

Matériel type: Holotype MNHN.F.A59993 (Coll. Turin). Paratype 1 spécimen (Coll. Turin).

Étymologie: l'espèce est dédiée à François Sirugue, ancien assistant du conservateur Raymond Dughi du Muséum d'Histoire Naturelle d'Aix-en-Provence.

Localité type: Vitrolles (Bouches-du-Rhône).

Étage : Rognacien supérieur (banc $\mathrm{n}^{\circ} 100$, Fig. 2).

Description: Lychnus siruguei nov.sp. est une coquille elliptique, légèrement carénée, ovale dans le sens du diamètre transversal, elle comporte cinq tours de spire, dont les tours juvéniles sont dissimulés sur la moitié par le dernier. Le sens d'inclinaison de ces jeunes tours reste oblique par rapport à l'axe général de la coquille, la hauteur des deux premiers 


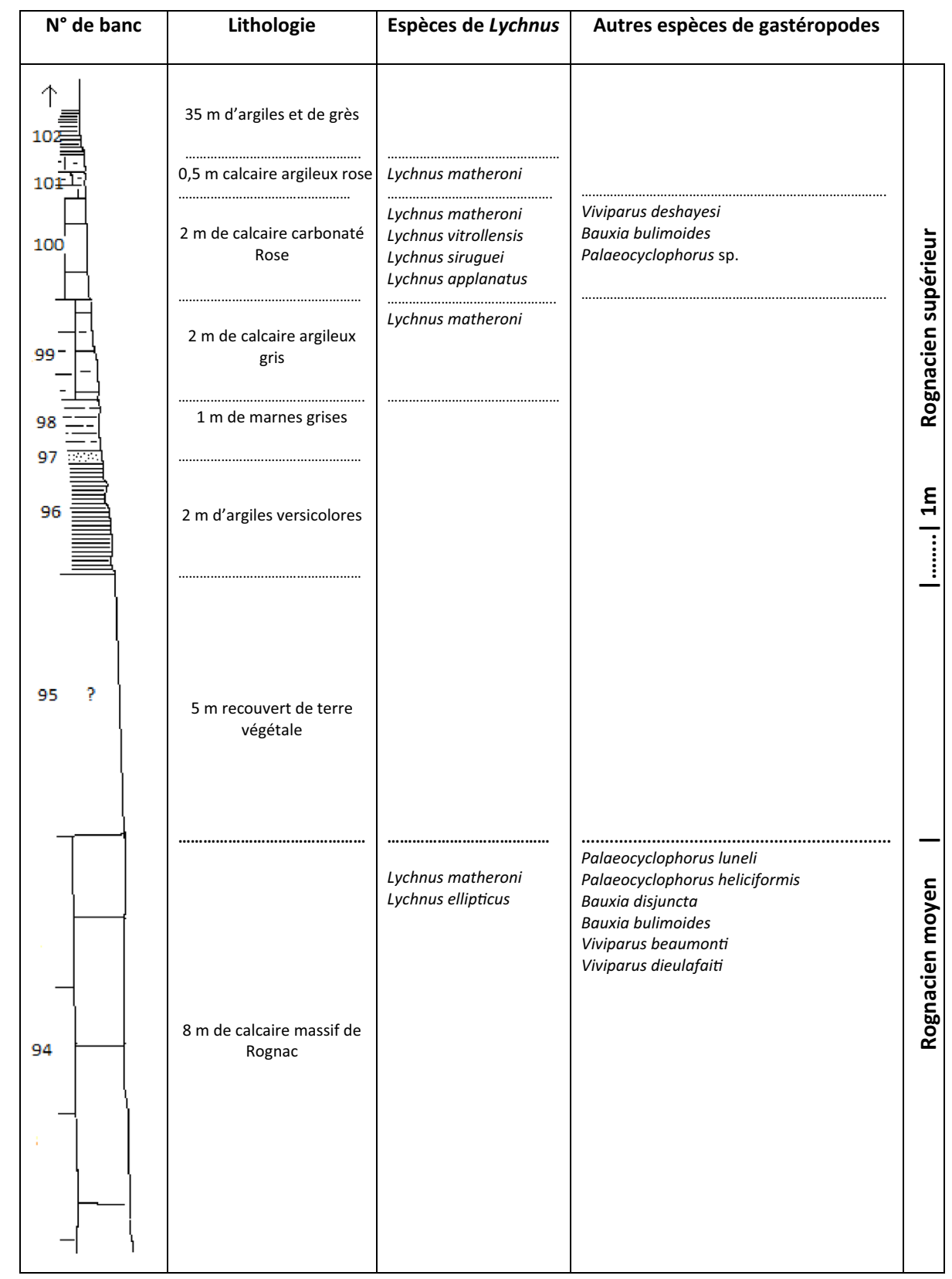

Fig. 2. Coupe A (Rognacien supérieur sous-jacent au radar de Vitrolles).

dépasse du reste de $2 \mathrm{~mm}$ l'épaisseur du tour final. La carène est plutôt subarrondie; elle divise la coquille en deux parties. La face supérieure nous permet d'observer que le dernier tour est peu recouvrant et l'inférieure ou ombilicale possède une ligne suturale en forme de demi-circonférence. Sur cette face inférieure, la terminaison du dernier tour reste moins large près de l'ouverture que vers le milieu. La face supérieure est ornée de fines stries d'accroissement flexueuses, mais le reste du test est lisse. L'ouverture reste ovale, elle est ceinturée par un péristome légèrement réfléchi.

Dimensions: diamètre longitudinal, $33 \mathrm{~mm}$; diamètre transversal, $35 \mathrm{~mm}$; hauteur, $14 \mathrm{~mm}$; diamètre de l'ouverture, $15 \mathrm{~mm}$; hauteur de la forme jeune, $15 \mathrm{~mm}$.
Rapports et différences: Lychnus siruguei nov. sp. se distingue des autres espèces carénées par un diamètre transversal supérieur au diamètre longitudinal. Dans l'ensemble, c'est à Lychnus matheroni qu'il ressemble le plus mais ce dernier est beaucoup plus imposant et comporte quatre tours de spire au total (Requien, 1842; Fritel, 1903), alors que $L$. siruguei nov. sp. en comprend cinq, dont quatre au stade juvénile. Cette espèce est la seule dans le genre Lychnus à avoir une dimension transversale supérieure à la longitude, quant à L. vitrollensis Répelin, 1920, son diamètre longitudinal ne dépasse pas les $30 \mathrm{~mm}$ et sa coquille comporte seulement quatre tours de spire. À propos de cette dernière espèce, je partage les mêmes opinions que Fabre-Taxy (1959) et 


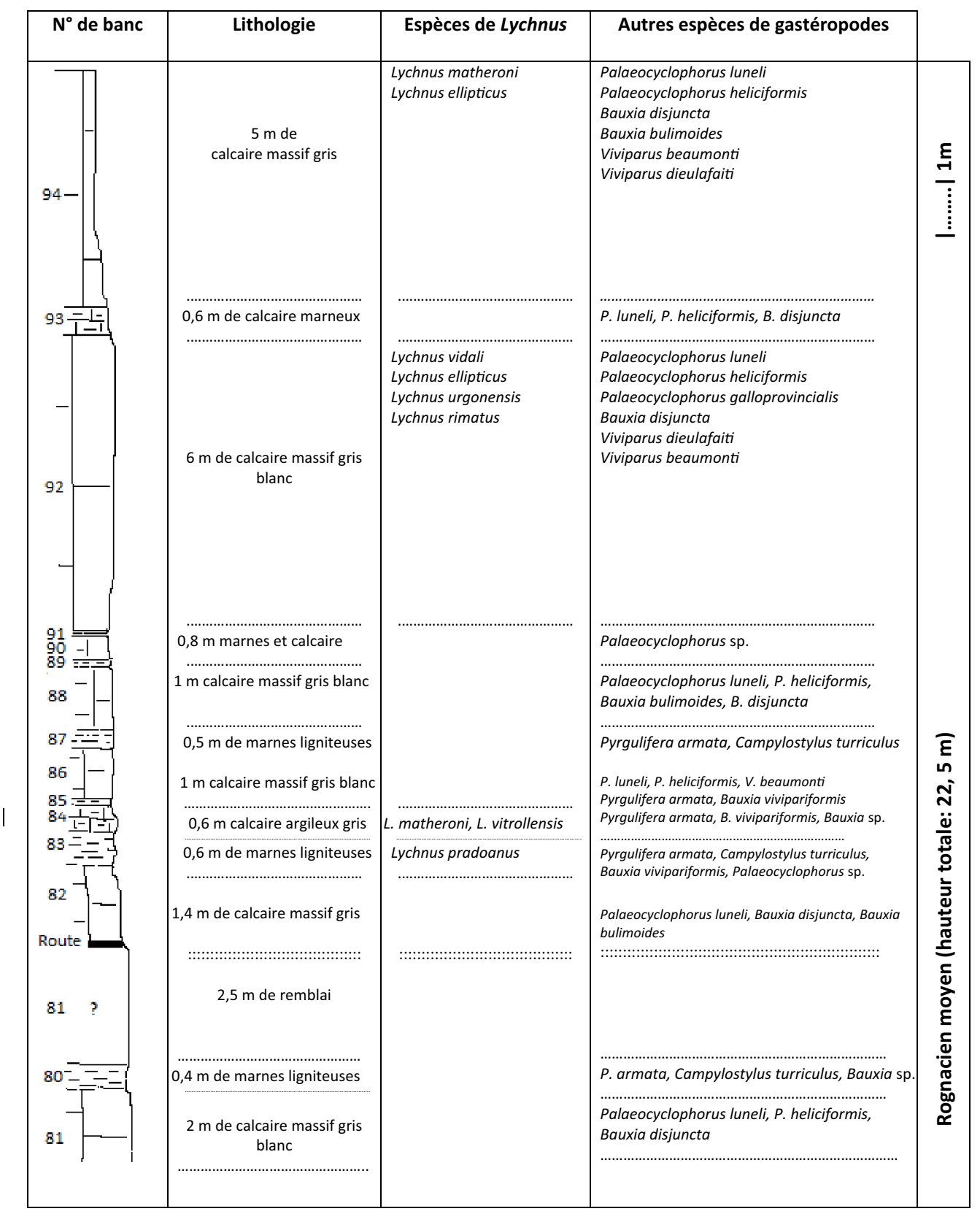

Fig. 3. Coupe B (Calcaire de Rognac sur la corniche du Roucas).

considère que L. vitrollensis est une sous-espèce de Lychnus matheroni, car ces deux espèces sont identiques à part les dimensions. Parmi toutes les espèces de ce genre, que ce soit des morphologies rondes ou carénées, Lychnus siruguei nov. sp. reste la seule qui comporte cinq tours pour une forme subovale. Un autre critère qui le différencie de L. matheroni reste l'aspect saillant dans le sens de la hauteur des deux premiers tours juvéniles. Les autres espèces provençales répertoriées dans le Rognacien, comprenant le même nombre de spires, sont les suivantes:L. dallonii Répelin, 1920, L. urgonensis et L. pradoanus Verneuil et Lartet, 1863. Lychnus dallonii comporte un sillon ombilical très long avec un diamètre longitudinal beaucoup plus important qui le différencie nettement de L. siruguei nov. sp., L. urgonensis se définit par une forme arrondie sur sa face supérieure et aplati sur le côté ombilical, celui-ci a des dimensions beaucoup plus importantes et enfin L. pradoanus qui comporte deux méplats circulaires autour de la carène.

Lychnus applanatus nov. sp. Pl., fig. E-G.

Matériel type : Holotype MNHN.F.A59994 (Coll. Turin). Paratype 1 spécimen (Coll. Turin).

Étymologie: la dénomination spécifique applanatus suggère la forme aplatie et étalée.

Localité type: Vitrolles (Bouches-du-Rhône). 


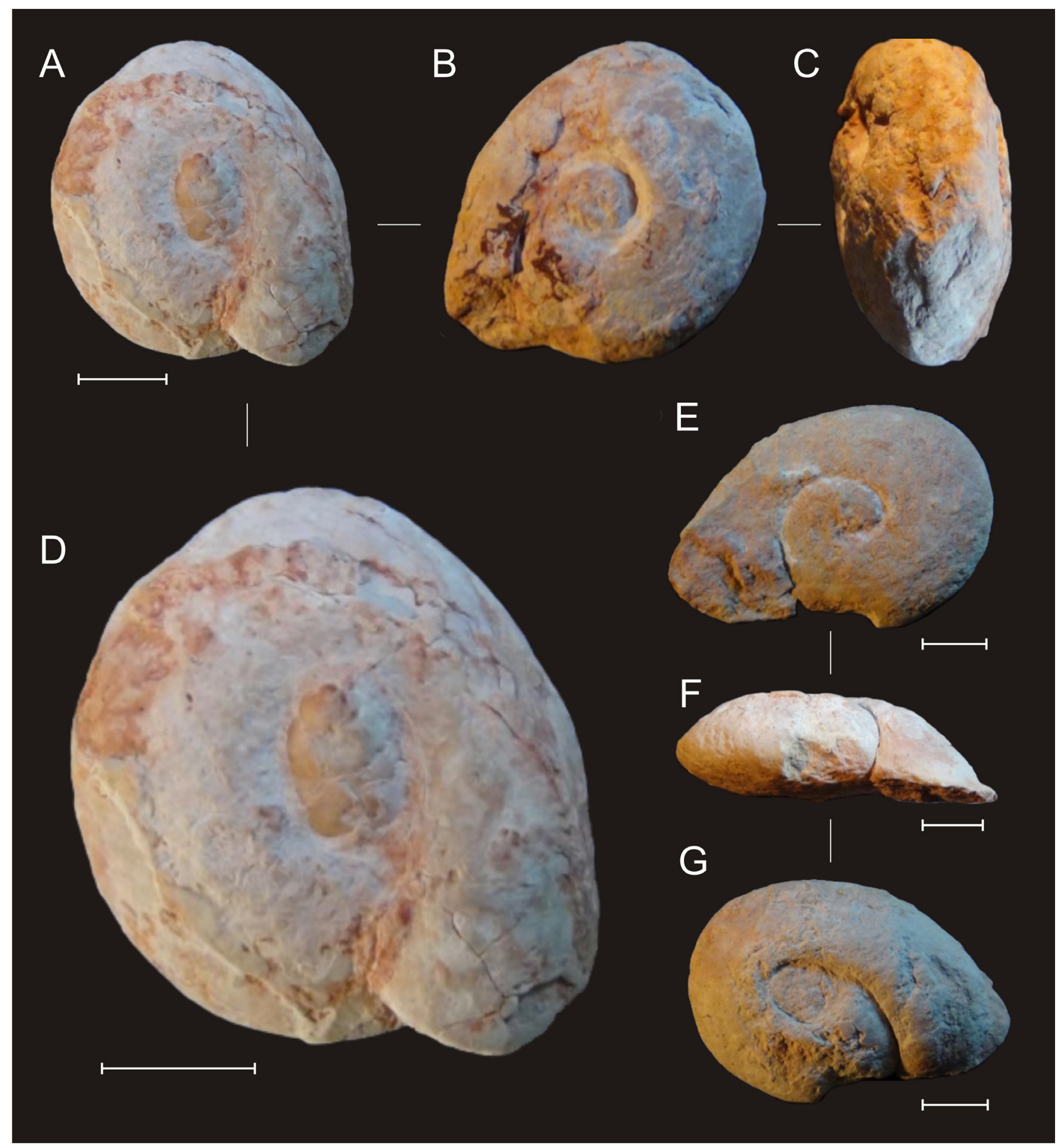

Planche 1. Lychnus provenant du Rognacien supérieur de Vitrolles, niveau 100 de la coupe A.

A-D. Lychnus siruguei nov.sp., holotype, MNHN.F.A59993 (Coll. Turin); A-C. Vues apicale, basale et abaperturale ; D. Grossissement de la vue apicale.

E-G. Lychnus applanatus nov. sp., holotype, MNHN.F.A59994 (Coll. Turin); vues apicale, abaperturale et basale.

Échelles : $10 \mathrm{~mm}$.

Étage : Rognacien supérieur (banc $n^{\circ} 100$, Fig. 2).

Description: Lychnus applanatus nov. sp. est une forme allongée et très étalée longitudinalement, la carène reste assez bien prononcée, elle divise la coquille en deux parties. La face supérieure est bombée, la terminaison du dernier tour avant l'ouverture présente un plan très oblique par rapport à l'axe de la coquille, quant à la face ombilicale, elle reste très aplatie. Les deux spécimens récoltés sont des moules internes, les tours 
juvéniles sont abrasés et ne permettent pas de déterminer leur nombre, dans l'ensemble, le dernier tour recouvre en épaisseur les premiers. La face inférieure, très comprimée, laisse apercevoir un sillon ombilical long et sinueux, le test reste lisse et les stries d'accroissement sont invisibles malgré la présence de coquille. L'ouverture reste ovale, elle est très écrasée sur la face ombilicale et ceinturée par un péristome réfléchi.

Dimensions : diamètre longitudinal, $50 \mathrm{~mm}$; d. transversal, $33 \mathrm{~mm}$; hauteur, $16 \mathrm{~mm}$; diamètre de l'ouverture longitudinale, $17 \mathrm{~mm}$; d. de l'ouverture transversale, $15 \mathrm{~mm}$.

Rapports et différences: cette espèce a beaucoup d'affinités avec Lychnus elongatus Roule, 1885, mais s'en distingue par ses dimensions plus importantes. En ce qui concerne la position stratigraphique, L. elongatus appartient à l'étage Valdonnien (Campanien) alors que L. applanatus nov. sp. ne se récolte que dans le Rognacien supérieur. Toutes les autres espèces de ce genre qu'elles soient des morphologies carénées ou rondes n'ont pas de ressemblance proche avec cette nouvelle forme.

\section{Répartition}

Ces deux nouvelles espèces proviennent uniquement du niveau calcaire du Rognacien supérieur de Vitrolles (banc ${ }^{\circ} 100$, Fig. 2). De cette localité type, elles sont connues chacune par deux exemplaires. Nous avons suivi cet horizon, au nord à Saragousse sur la commune de Rognac et au sud sur le Griffon, mais en dépit de plusieurs années de recherches, nous n'y avons jamais trouvé ni L. siruguei nov. sp., ni L. applanatus nov. sp.

Les deux autres espèces, Lychnus matheroni, et $L$. vitrollensis recensées également dans ce banc $\mathrm{n}^{\circ} 100$, sont présentes dans les horizons ligniteux intermédiaires au calcaire de Rognac. Ces deux espèces se rencontrent aussi dans le niveau $\mathrm{n}^{\circ} 84$ de ce calcaire, au niveau de la corniche du Roucas (Fig. 3).

Nous n'avons pas négligé de prospecter dans le calcaire de Rognac et ses niveaux ligniteux sur la commune des PennesMirabeau. Ces prospections ont été menées jusqu'à la sortie du tunnel de l'autoroute A7. Dans un banc marneux de ce Rognacien moyen, un exemplaire de L. matheroni et de Lychnus cf. gardanensis Matheron in Répelin, 1920.

\section{Conclusion}

Les espèces Lychnus siruguei nov. sp. et L. applanatus nov. sp. apparaissent à Vitrolles au sommet du banc $n^{\circ} 100 \mathrm{du}$ Rognacien supérieur. Elles coexistent avec L. matheroni et $L$. vitrollensis avant de disparaître complètement avec les autres gastéropodes dans les niveaux d'argiles et calcaire marneux sus-jacent, qui sont encore Rognacien. Seule l'espèce $L$. matheroni a été encore récoltée récemment dans le banc $n^{\circ} 101$ (Fig. 2). Ce taxon sera le dernier représentant du genre, l'extinction définitive des Lychnus sera enregistrée dans la zone supérieure (niveau $\mathrm{n}^{\circ} 102$ ).
La découverte de ces nouvelles espèces rarissimes qui sont peut-être des taxons endémiques renforce l'originalité paléobiogéographique des faunes de Lychnus de Provence. Pour la même période, ce genre qui est également présent en Espagne est moins diversifié.

En recensant ces deux nouvelles formes, ce sont treize espèces du genre Lychnus qui sont désormais connues dans le Rognacien des Bouches-du-Rhône et quinze au total en ajoutant les sous étages Bégudien et Valdonnien (Campanien).

Remerciements. Je tiens à remercier tout particulièrement Jean-Luc Martin pour sa contribution sur le terrain et Régis Martin qui a conservé l'intégralité des exemplaires de gastéropodes recueillis.

\section{Références}

Dughi R, Sirugue F. 1958. Sur les œufs de dinosaures du bassin fluviolacustre de Basse-Provence. Section des sciences, $83^{\circ}$ congrès des sociétés savantes. p. 192.

Durand JP. 1978. Notice explicative de la Feuille de MartiguesMarseille à 1/50 000. Édition du Bureau de Recherches Géologiques et Minières, Orléans, pp. 1-51.

Fabre-Taxy S. 1959. Faunes lagunaires et continentales du Crétacé supérieur de Provence. Le Maestrichtien et le Danien. Annales de Paléontologie 45: 55-124.

Fritel PH. 1903. Histoire Nat. de la France, $24^{\circ}$ partie, Paléontologie. Deyrolle, Paris, $229 \mathrm{p}$.

Linnaeus C. 1753. Species plantarum, exhibentes plantas rite cognitas, ad genera relatas, cum differentiis specificis, nominibus trivialibus, synonymis selectis, locis natalibus, secundum systema sexuale digestas, vol. 2. Impensis Laurentii Salvii, Holmiae, Stockholm, p. [i], 561-1200, [1-30, index], [i, err.].

Matheron P. 1832. Observations sur les terrains tertiaires des Bouches-du-Rhône et description des coquilles fossiles inédites ou peu connus qu'ils renferment. Annales des Sciences et Industrie du Midi de la France 3: 1-87.

Matheron P. 1842. Catalogue méthodique et descriptif des corps organisés fossiles du département des Bouches-du-Rhône et lieux circonvoisins. Répertoire des travaux de la société de statistique de Marseille 6 (81-342): 1-41.

Munier-Chalmas E. 1885. Miscellanées paléontologiques. Annales de Malacologie 1: 323-339.

Repelin J. 1920. Monographie du genre Lychnus. Mémoires de la société géologique de France. Paléontologie 53 (1-24): 1-6.

Requien E. 1842. Description du Lychnus matheroni. Bulletin de la société géologique de France 13: 495-496.

Roule L. 1885. Description de quelques coquilles fossiles du calcaire lacustre de Rognac (Bouches-du-Rhône). Bulletin de la société malacologique 1: 311-326, pl. VII-VIII.

Verneuil E. de, Lartet L. 1863. Note sur le calcaire a Lychnus des environs de Segura (Aragón). Bulletin de la société géologique de France 20: 684-698.

Wenz W. 1940. Die ältesten Stylommatophoren des europaischen Raumes. Archiv für Molluskenkunde 72: 129-144.

Citation de l'article : Turin G. 2017. Description de deux espèces nouvelles de Lychnus (Mollusca, Gastropoda, Anadromidae) du Rognacien (Maastrichtien supérieur) de Vitrolles (Bouches-du-Rhône, France), BSGF - Earth Sciences Bulletin 188: 38. 